\title{
Simulation of Spatial Variations of RF Exposure Within a Macrocell
}

\author{
D. Plets, W. Joseph, L. Martens \\ Information Technology Department, Ghent University/iMinds \\ Technologiepark 15, B-9052 Ghent, Belgium \\ david.plets@intec.ugent.be
}

\begin{abstract}
The spatial variations of the time-averaged total whole-body SAR due to voice calls within a $3 G$ macrocell are investigated by simulations. The impact of cell size, usage intensity, usage environment and user morphology is investigated. It is shown that within a cell, total SAR values are minimal when uplink exposure equals downlink exposure. Average exposure increases when the cell size increases, when calls are longer and executed indoor, and when executed by children.
\end{abstract}

\section{INTRODUCTION}

Concerns about a possible health impact of radio-frequency (RF) electromagnetic fields have led to international regulations and limitations (compliance studies), but also characterization of real-life exposure has been receiving a lot of research attention. Recently, the Exposure Index [1] has been formulated as a way to quantify the average exposure of humans in daily life. In this paper, it is investigated how the total (uplink (UL) + downlink (DL)) individual Specific Absorption Rate (SAR) varies spatially within the serving macrocell, and how this distribution is impacted by altering the (UL) usage intensity, the cell size, environment of the specific location (indoor vs. outdoor), and the morphology of the user (child vs. adult).

\section{Methodology}

Simulations are done for a $3 \mathrm{G}$ macrocell base station (MBS) network, that is designed for indoor phone call coverage. Its range is calculated based on the following assumptions: the COST231 Walfisch-Ikegami model is used, an average building penetration loss of $8 \mathrm{~dB}[2]$ (standard deviation $\sigma=6 \mathrm{~dB}$ ), a shadowing margin of $10 \mathrm{~dB}$, and a fading margin of $9 \mathrm{~dB}$ are assumed. The sensitivities of the mobile user device and MBS are assumed at $-110 \mathrm{dBm}$ and $-118 \mathrm{dBm}$ respectively. Whole-body reference SAR values are $4.6 \mathrm{~mW} / \mathrm{kg}$ per $\mathrm{W} / \mathrm{m}^{2}$ for DL and $5.2 \mathrm{~mW} / \mathrm{kg}$ per $\mathrm{W}$ for UL [3].

Three scenarios are compared to a reference scenario, which is defined as follows: a macrocell with a serving base station operating with an Effective Isotropically Radiated Power (EIRP) of $38 \mathrm{dBm}$, for outdoor users calling during $35 \mathrm{~s}$ per hour on average. Each of the three scenarios will investigate the impact of a change with respect to the reference scenario. In Scenario 1, the cell size will be changed by altering the EIRP to $30 \mathrm{dBm}$ and to $46 \mathrm{dBm}$. In Scenario 2, usage will be considered indoor (assuming a building penetration loss of $8 \mathrm{~dB}$ ) instead of outdoor. In Scenario 3, the phone call intensity (PCI) will be altered to light ( $8.75 \mathrm{~s}$ per hour) and to high (140 s/h). In Scenario 4, child users will be assumed with whole-body reference SAR values of $7.7 \mathrm{~mW} / \mathrm{kg}$ per $\mathrm{W} / \mathrm{m}^{2}$ for $\mathrm{DL}$ and $10.0 \mathrm{~mW} / \mathrm{kg}$ per $\mathrm{W}$ for UL [3]. For each of the scenarios, the global average whole-body SAR within the cell will be assessed and it will be investigated how the total individual time-averaged whole-body SAR spatially varies within the cell. This analysis will be done for the reference scenario and a comparison with each of the four defined scenarios will be made.

\section{RESULTS}

Figures 1 - 4 show a comparison of the reference scenario (denoted with $\mathrm{R}$ in the figures) with Scenarios 1 to 4 respectively, each showing the DL, UL, and total (DL+UL) exposure within the cell range as well as the average exposure within the cell. All figures show that a minimal total SAR (red lines) is observed where UL and DL exposure are equal. This is due to the quadratic decrease (increase) of the DL (UL) exposure as a function of the distance.

Fig1(Scenario 1) shows that for higher cell sizes (higher EIRP of the MBS), the DL exposure increases and the location with a minimal SAR is further from the MBS, as the DL exposure is lower there. Globally, the SAR is higher for larger cells, due to the increased DL power density at smaller distances from the MBS, and the increased UL power at the edges of the (larger) cell (cell average of $3.6 \cdot 10^{-9}, 1.7 \cdot 10^{-8}$, and $9.6 \cdot 10^{-8} \mathrm{~W} / \mathrm{kg}$ for 30,38 and $46 \mathrm{dBm}$, respectively).

Fig. 2 (Scenario 2) shows that for indoor users, the minimal SAR remains the same, but its location is closer (at $52 \mathrm{~m}$ vs. at $85 \mathrm{~m}$ ) to the $\mathrm{MBS}$, as the DL exposure lowers and UL exposure increases at each location, by a factor that is equal to the assumed penetration loss. Globally, the SAR increases for indoor usage, due to the fact that UL exposure, which is dominant over the largest part of the cell, becomes higher indoors. The cell average increases from $1.7 \cdot 10^{-8} \mathrm{~W} / \mathrm{kg}$ to $8.9 \cdot 10^{-8} \mathrm{~W} / \mathrm{kg}$ when using indoor instead of outdoor, corresponding to a factor 5.1 or $7.1 \mathrm{~dB}$. This is indeed close to the assumed penetration loss of $8 \mathrm{~dB}$, indicating the dominance of the UL contribution in the total exposure.

From Fig. 3 (Scenario 3), it is observed that the higher the PCI, the higher the UL contribution, and therefore, the closer to the MBS the location with a minimal SAR. Logically, the averaged SAR over the entire cell increases as the PCI 


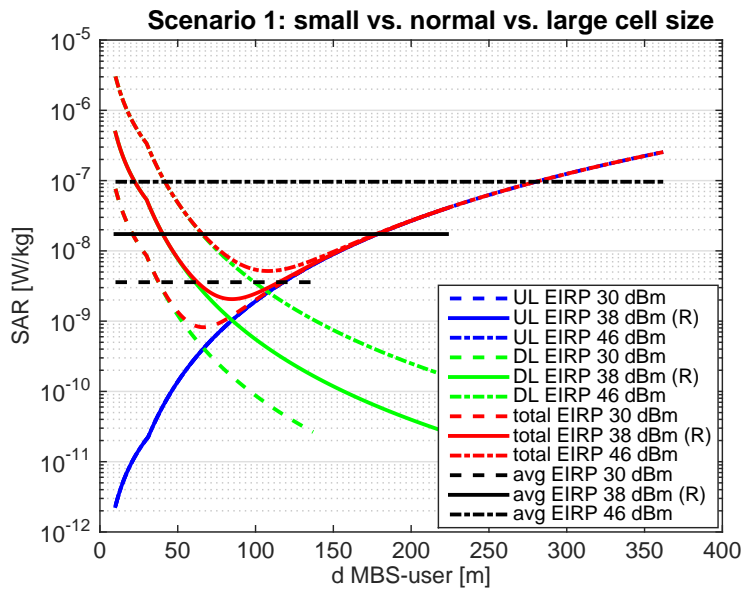

Fig. 1. Spatial distribution of SAR $[\mathrm{W} / \mathrm{kg}]$ in Scenario $1(\mathrm{R}=\mathrm{ref})$.

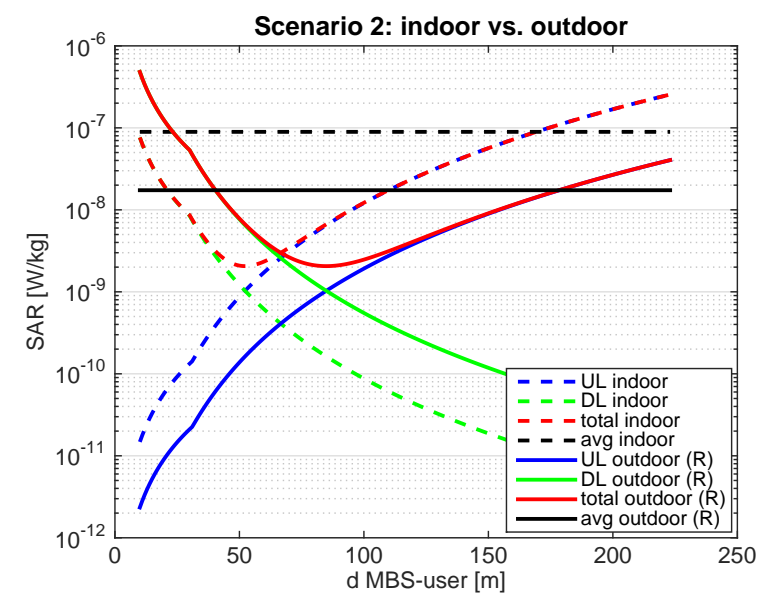

Fig. 2. Spatial distribution of SAR $[\mathrm{W} / \mathrm{kg}]$ in Scenario 2 (R=ref).

increases, e.g., by a factor 3.4 when switching from normal $(35 \mathrm{~s} / \mathrm{h})$ to high $(140 \mathrm{~s} / \mathrm{h})$ usage.

Finally, Fig. 4 (Scenario 4) shows that for child users, the minimal-exposure location is more or less the same compared to adult users (adult at $85 \mathrm{~m}$ vs. child at $83 \mathrm{~m}$ ), since the ratio of the UL and DL reference SARs for children vs. adults are comparable. The average SAR over the cell increases by $87 \%$, due to the higher (UL and DL) reference SAR values for children, with a maximum increase of $92 \%$ at the cell edge.

\section{CONCLUSIONS}

Spatial variations of the whole-body SAR within a macrocell are investigated, as well as cell-averaged exposure values. Minimal exposure values are observed when UL and DL SAR become equal. Cell-averaged exposure increases for larger cell sizes (a factor 5.5 when the base station EIRP increases from 38 to $46 \mathrm{dBm}$ or the cell range from 223 to $362 \mathrm{~m}$ ), when usage duration increases (by a factor 3.4 when usage duration is quadrupled), and when this usage happens indoor (by a factor 5.2). It is also shown that exposure of children is up to

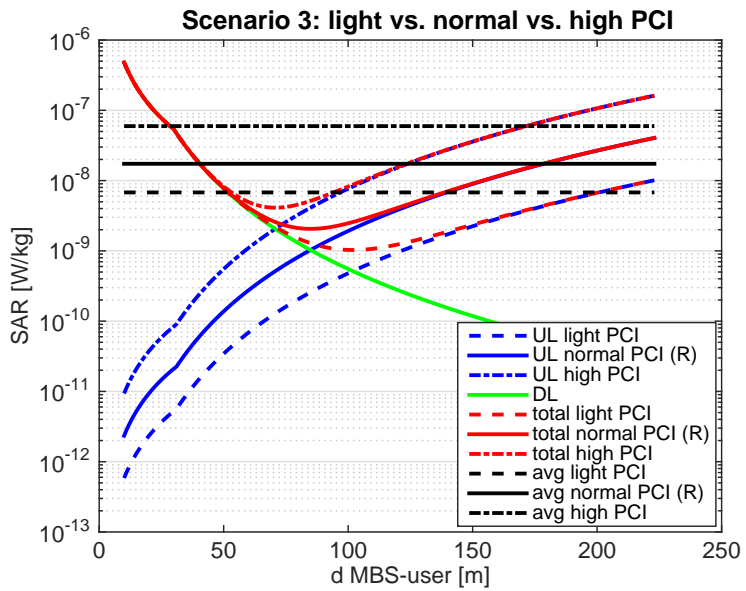

Fig. 3. Spatial distribution of SAR $[\mathrm{W} / \mathrm{kg}]$ in Scenario 3 (R=ref).

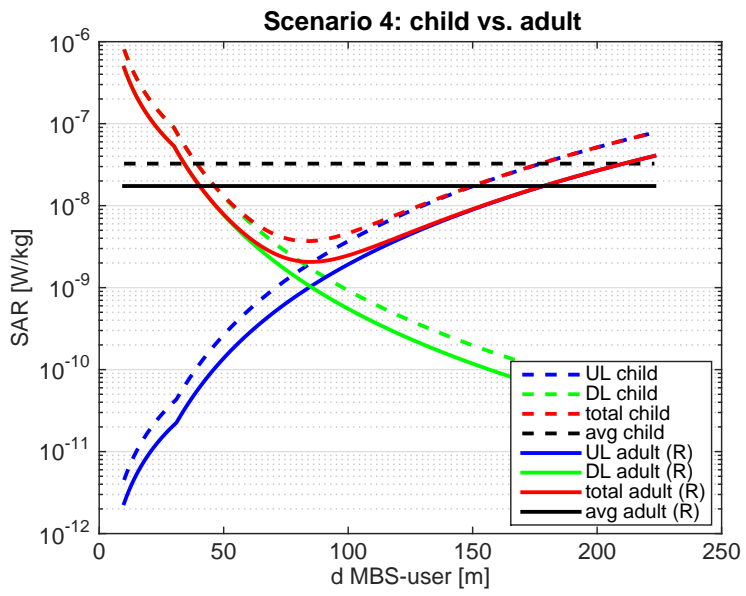

Fig. 4. Spatial distribution of SAR $[\mathrm{W} / \mathrm{kg}]$ in Scenario 4 (R=ref).

92\% larger than for adults. The results show that exposure due to uplink traffic mostly dominates exposure due to downlink traffic, indicating the possible benefits of using femtocells.

\section{ACKNOWLEDGEMENT}

This paper reports work undertaken in the context of the project LEXNET, a project supported by the European Commission in the 7th Framework Programme (GA no318273). For further information, please visit www.lexnet-project.eu.

\section{REFERENCES}

[1] Varsier, N., Plets, D., Corre, Y., Vermeeren, G., Joseph, W., Aerts, S., Martens, L. and Wiart, J., "A novel method to assess human population exposure induced by a wireless cellular network." BioElectromagnetics, vol. 36, pp. 451-463, 2015.

[2] D. Plets, W. Joseph, L. Verloock, E. Tanghe, L. Martens, H. Gauderis, and E. Deventer, "Extensive Penetration Loss Measurements and Models for Different Building Types for DVB-H in the UHF Band," IEEE Transactions on Broadcasting, vol. 55, no. 3, pp. 213-222, 2009.

[3] Lexnet consortium, "Lexnet D2.8: Global Wireless Exposure Metric Definition," Tech. Rep., http://cordis.europa.eu/docs/projects/cnect/3/318273/080/deliverables/001LEXNETWP2D28GlobalwirelessexposuremetricdefAres20155347928.pdf. 